\title{
Therapeutics
}

\section{Hyaluronic acid improved walking ability and short term knee function in osteoarthritis of the knee}

Huskisson EC, Donnelly S. Hyaluronic acid in the treatment of osteoarthritis of the knee Rheumatology 1999 Jul;38:602-7.

QUESTION: In patients with osteoarthritis of the knee, is hyaluronic acid (HA) effective for relieving symptoms?

\section{Design}

Randomised (unclear allocation concealment*), blinded (outcome assessor and $\{$ patients $\} \uparrow$ ), placebo controlled trial with 6 month follow up.

\section{Setting}

A hospital outpatient clinic in London, UK.

\section{Patients}

100 patients (mean age $65 \mathrm{y}, 67 \%$ women) who had osteoarthritis of 1 or both knees according to the criteria of the American Rheumatism Association, changes in osteoarthritis (Kellgren and Lawrence grade II or III) on radiography in the previous 6 months, consistent pain in the previous 3 months, and moderate or severe pain on walking. Exclusion criteria were grade IV changes on radiography, functional impairment of the knee, associated osteoarthritis of any other joint that might affect assessment of the knee, psoriasis, other joint diseases or infection, other painful knee conditions, serious medical illness, skin conditions overlying the joint, or use of intra-articular steroids or radiocolloids in the previous 3 months. Follow up at 6 months was $81 \%$.

\section{Intervention}

Patients were allocated to HA, $20 \mathrm{mg}$ in $2 \mathrm{ml}$ of aqueous solution $(n=50)$, or to placebo $(n=50)$ given in 5 weekly intra-articular injections.

\section{Main outcome measures}

Knee pain on walking (100 mm visual analogue scale) and knee function (Lequesne Functional Index).

\section{Main results}

Patients in the HA group had less pain on walking at 5 weeks $(p=0.009)$ and 6 months $(p=0.005)$ than did patients in the placebo group (table). Knee function was more improved in the HA group at week 5 than in the placebo group $(\mathrm{p}=0.04)$; the difference persisted to 4 months (table).

\section{Conclusion}

In patients with osteoarthritis of the knee, hyaluronic acid was effective for reducing pain on walking at 6 months and improving knee function for 4 months.

*See glossary.

$\dagger$ Information supplied by author.

\section{COMMENTARY}

Management of symptomatic osteoarthritis includes nonsteroidal anti-inflammatory drugs (NSAIDs), intra-articular corticosteroids, viscosupplementation, and ultimately total knee arthroplasty. In a well executed, randomised, placebo controlled trial, Huskisson and Donnelly have shown that viscosupplementation with a course of 5 weekly HA (Hyalgan) injections can relieve pain for 6 months and improve function for 4 months in relatively healthy patients with a mean age of 66 years. This study showed that the beneficial effect is at least as good as that achieved with NSAIDs but without the gastrointestinal side effects.

Whereas Hyalgan requires 5 injections, ${ }^{1}$ Hylan G-F 20 (Synvisc), another viscosupplementation agent, is effective after 3 weekly injections. ${ }^{2}$ Findings show that courses of Synvisc injections can be repeated every 6 months and may thus provide several years of relief.' The study by Huskisson and Donnelly showed that injection site pain and local reactions may occur in up to $30 \%$ of patients receiving Hyalgan, but such adverse effects are also common with placebo injections. ${ }^{1}$ The lateral approach with a straight knee results in the fewest problems.

The precise indications for viscosupplementation remain somewhat controversial, although patients with early arthritis and moderate symptoms seem to gain the most benefit from this intervention.

Hans J Kreder, MD, MPH University of Toronto and Sunnybrook and Women's College Health Sciences Centre Toronto, Ontario, Canada

1 Altman RD, Moskowitz R. Intraarticular sodium hyaluronate (Hyalgan) in the treatment of patients with osteoarthritis of the knee: a randomized clinical trial. Hyalgan Study Group. $J$ Rheumatol 1998;25:2203-12.

2 Adams ME, Atkinson MH, Lussier AJ, et al. The role of viscosupplementation with Hylan G-F 20 (Synvisc) in the treatment of osteoarthritis of the knee: a Canadian multicenter trial comparing Hylan G-F 20 alone, Hylan G-F 20 with non-steroidal anti-inflammatory drugs (NSAIDs) and NSAIDs alone. Osteoarthritis Cartilage 1995;3:213-25.

3 Lussier A, Cividino AA, McFarlane CA, et al. Viscosupplementation with hylan for the treatment of osteoarthritis: findings from clinical practice in Canada. I Rheumatol 1996;23:1579-85

Source of funding: no external funding.

For correspondence: Dr S Donnelly, Department of Rheumatology, Whipps Cross Hospital, London E11 1NR, UK. Fax $+44(0) 2085588115$.

Hyaluronic acid (HA) v placebo for osteoarthritis of the knee

\begin{tabular}{llll}
\multirow{2}{*}{$\begin{array}{l}l \\
\text { Outcomes }\end{array}$} & Mean scores & Mean score difference \\
\cline { 2 - 4 } & HA & Placebo & (95) $\neq$
\end{tabular}

$\ddagger$ Mean score difference and $\mathrm{Cl}$ calculated from data in article; differences favour the $\mathrm{HA}$ group. 